\title{
A quantitative thermal wave assessment of the characteristics of sub-surface defects
}

by D.P. ALMOND and P.M. PATEL $(*)$

\author{
(") School of Materials Science, University of Bath, Bath, BA2 7AY, UK.
}

\begin{abstract}
The thermal wave characteristics of well characterised air-gap defects are compared with the predictions of one-dimensional heat flow theory. It is found that the lateral dimensions significantly affect thermal wave response. A first order finite defect model is presented which accounts for much of the response obtained from real finite defects.
\end{abstract}

\section{Introduction}

There have been numerous demonstrations of the ability of thermal wave techniques to detect the presence of sub-surface defects or features. As thermal wave techniques are now being seriously considered for use in NDE applications, it is important that they are subjected to a full quantitative assessment. Issues of interest include: the intrinsic resolving power of the technique; the effect of defect air-gap thickness; the influence of defect depth and the nature of the host material. In addition, there is evidence that parameters such as defect depth and air-gap thickness can be obtained from a quantitative assessment of the defect's thermal wave response.

In this paper experimental data from well characterised defects are presented which underline the necessity of developing a full three-dimensional heat flow analysis to properly characterise defect responses.

\section{Experimental}

Measurements were made using a photothermal testing system in which thermal waves were generated by a modulated laser and detected with an infrared detector. The two testpieces illustrated in figure 1 were investigated. One (figure 1a) was a block of mild steel which contained a $5 \mathrm{~mm}$ and a $10 \mathrm{~mm}$ diameter (air-gap thickness $100 \mu \mathrm{m}$ ) artificial defect 1 $\mathrm{mm}$ beneath the surface. The second test-piece (figure $1 \mathrm{~b}$ ) contained an adhesion defect at the interface between a plasma sprayed aluminium coating and a mild steel substrate. The defective area was a $20 \mu \mathrm{m}$ air-gap in the form of a strip $3 \mathrm{~mm}$ wide across the test-piece.

Measurements were taken of the change in thermal wave phase caused by the presence of the defects over a range of modulation frequencies from 4 to $160 \mathrm{~Hz}$.

\section{One-dimensional air-gap analysis.}

It has been shown before [1] that a simple layer model may be used to determine the effects of air-gap defects on thermal wave response. In this model the effective reflection coefficient of the air-gap is calculated and it is this which is found to depend on the gap thickness, the modulation frequency and the relative thermal properties of the host material. This reflection coefficient is then inserted in the well tried thermal wave interference expressions [2] to obtain the nett effect of the defect. 


\section{http://dx.doi.org/10.21611/qirt.1992.057}

The experimental results are compared in figures 2 and 3 with the predictions of the onedimensional layer model employing the expressions that were presented before [1]. The results obtained from the $10 \mathrm{~mm}$ diameter defect in the mild steel test-piece are in excellent agreement with this model, indicating the air-gap to have the correct thickness of about 100 $\mu \mathrm{m}$. Those for the $5 \mathrm{~mm}$ diameter defect, however, would seem to be greatly in error as they indicate a gap thickness of about $3 \mu \mathrm{m}$, whilst this defect also had a gap thickness of about $100 \mu \mathrm{m}$. Similarly, the results for the strip defect beneath the aluminium coating depart very significantly from the model predictions for a $20 \mu \mathrm{m}$ air-gap defect. The differences between the 5 and $10 \mathrm{~mm}$ diameter results, figures $2 b$ and $2 a$, provide a particularly clear example of the ways in which the lateral extent of a defect affects its thermal wave characteristics in the frequency domain and how these could lead to very significant errors in the assessment of the thickness of an air gap.

\section{Finite size defect response.}

A first-order approximation to the effects that defect size has on its frequency domain response can be obtained by treating a circular defect as a perfectly reflecting disk. The thermal wave response of this at the surface, over its centre, may be obtained employing a simple Huygens construction [3]. The resulting expression for a defect of radius $a$ at a depth $z$ in a medium having thermal conductivity $k$ is:

$$
T(a, z)=\frac{1}{k \sigma}+\frac{2 \exp (-\sigma z)}{k \sigma}\left[\exp (-\sigma z)-\frac{z}{\sqrt{a^{2}+z^{2}}} \exp \left(-\sigma \sqrt{a^{2}+z^{2}}\right)\right]
$$

in which $\sigma$ is the thermal wave number. The data obtained from the $5 \mathrm{~mm}$ diameter defect are compared with the predictions of this expression in figure 4 . The agreement with the data is good and it is clear that this finite defect analysis explains much of the departure from the predictions of one- dimensional layer theory. Small defects (a few thermal diffusion lengths in dimension) exhibit a reduced reflection coefficient and, consequently, a response like that of a weakly reflecting, narrow air-gap. The Huygens construction makes the physical origins of this clear since only those secondary wave sources on the defect contribute to the reflection rather than all those in the defect plane, as effectively assumed in the one-dimensional analysis.

It is recognised that treating the defect as a uniformally reflecting disk is very much a first approximation and one which fails to satisfy boundary conditions at the disk edges. A rigorous analysis of the edge effects, which will be the subject of a future publication, shows the temperature on the disk to fall to zero at its edge over a distance of the order of the thermal diffusion length, rather than dropping abruptly to zero at the edge as assumed here. The nett effect is to further reduce the reflecting power of the defect and to sharpen its image at the surface. These effects will be strongest at lower modulation frequencies where the thermal wave length becomes an increasingly large fraction of the defect diameter.

\section{Conclusions}

It has been shown that, although in principle the thickness of the air-gap in a defect can be determined from its frequency domain thermal wave response, full account must also be taken of the lateral extent of the defect. Whilst this study has concentrated on examples in which the defect has taken the form of an air-gap, exactly the same conclusions would be drawn about defects that are better represented by a thermal contact resistance.

\section{Acknowledgements}

The authors are grateful to the Science and Engineering Research Council for their support of this work. 


\section{REFERENCES}

[1] PATEL (P.M), ALMOND (D.P) and REITER (H).-Thermal wave detection and characterisation of sub-surface defects. Appl. Phys. B 43, 1987, p9-15.

[2] BENNET (C.A) and PATTY (R.R).-Thermal wave interferometry: a potential application of the photoacoustic effect. Appl. Optics 21, 1982, p49-54.

[3] GREEN (G).-On a fundamental problem in diffraction. Phil. Mag.21, 1936, p934-947.
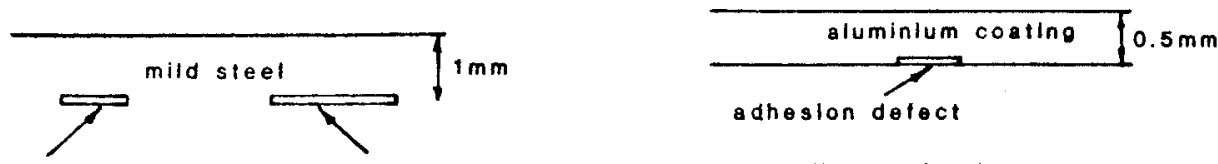

$5 \mathrm{~mm} \mathrm{defect}$

$10 \mathrm{~mm}$ defect

a

b

Fig. 1 - Schematic dlagrams of the defects.
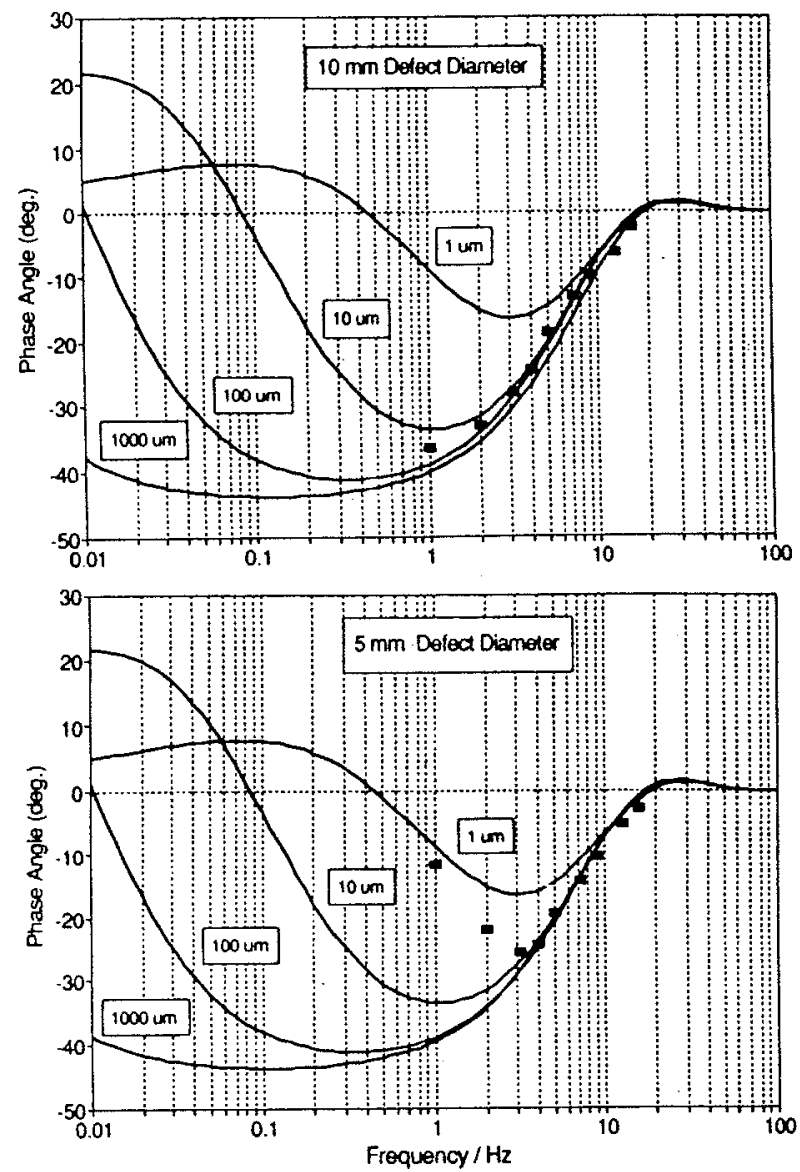

Fig. 2 - Frequency dependences of the thermal wave phase changes measured over the centres of (a) the $10 \mathrm{~mm}$ diameterdefect and (b) the $5 \mathrm{~mm}$ diameter defect compared with the predictions of onedimensional defect theory for air-gaps of thicknesses indicated. 
http://dx.doi.org/10.21611/qirt.1992.057

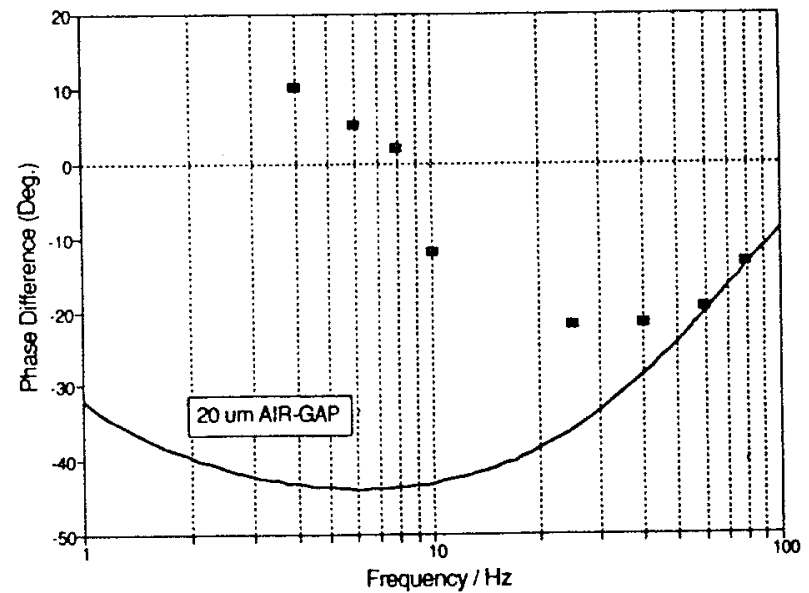

Fig. 3 -- Frequency dependence of the thermal wave phase change over the strip defect beneath the aluminlum coating compared with predictions of one - dimensional defect theory for a $20 \mu \mathrm{m}$ alr-gap.

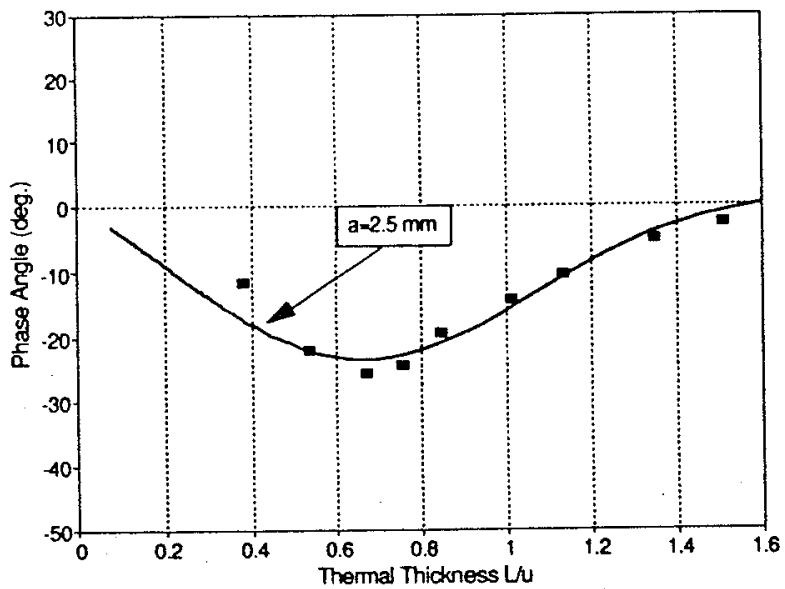

Fig. 4 - The data, flg. $2 b$, for the $5 \mathrm{~mm}$ dlameter defect compared with the predictions of the finite defect model. 